\title{
Concomitant appearance of intrinsic thermotolerance and storage of trehalose in Saccharomyces cerevisiae during early respiratory phase of batch-culture is CIF1- dependent
}

\author{
Paul V. Attfield, Sophia Kletsas and Brian W. Hazell
}

Author for correspondence: Paul V. Attfield. Tel: +612887 1911. Fax: +6128883178.

Yeast Physiology Research Group, Burns Philp Technology and Research Centre, PO Box 219, 67 Epping Road, North Ryde, New South Wales 2113 Australia

\begin{abstract}
Strains of Saccharomyces cerevisiae that exhibit varied capacities for accumulation of trehalose were tested for intrinsic thermotolerance. Yeast that accumulated trehalose rapidly in early respiratory phase showed equally rapid attainment of thermotolerance, whereas a strain unable to accumulate trehalose at this stage of culture showed markedly delayed appearance of thermotolerance. These results were obtained using closely related but nonisogenic diploids and so it is possible that variable factors other than trehalose were responsible for the observed thermotolerance effects. Therefore, a pair of isogenic diploid S. cerevisiae strains was generated to facilitate further testing of whether trehalose functions in intrinsic stress tolerance. Both isogenic strains inherited a partially reverted cif1 phenotype, designated CPR, from the trehalose-deficient progenitor that had been used in construction of the non-isogenic strains. The CPR phenotype permitted growth on glucose but not accumulation of trehalose, indicating that not all cif1-related deficiencies were suppressed in the CPR strains. However, one of the isogenic CPR pair was cif1/cif1 and failed to accumulate trehalose, whilst the other was cif1/CIF1 and was able to accumulate this sugar. The trehalose-proficient strain showed intrinsic stress tolerance whereas the trehalose-deficient strain was sensitive to heat stress during early respiratory growth. These results suggest that one or more functions of CIF1, not operating in the cif1/cif1(CPR) strains, are important for intrinsic thermotolerance of yeast in early respiratory phase. When considering these results with those of others whose work has indicated a role for trehalose in protection of proteins and membranes, it is reasonable to hypothesize that the trehalose deficiency associated with cif1/cif1(CPR) strains could be a key factor in their intrinsic thermosensitivity. However, if this is the case the importance of trehalose, relative to other stress tolerance factors, appears to vary with growth phase and culture status.
\end{abstract}

Keywords: Saccharomyces cerevisiae, growth phases, trehalose, thermotolerance, stress response

\section{INTRODUCTION}

The yeast Saccharomyces cerevisiae exhibits several distinct phases of growth when cultured on glucose in aerobic batch-culture (for example, see Lewis et al., 1993). There are two separate periods of exponential growth. The first and most rapid of these, termed respiro-fermentative, involves mainly fermentative metabolism of glucose to form alcohol, whereas the second exponential growth phase, termed respiratory, produces a slower growth rate and involves oxidative metabolism of the accumulated ethanol. Stress tolerance of $S$. cerevisiae varies greatly with physiological state and cultural conditions. For example, cells growing rapidly are more sensitive to stresses such as heat and freezing (Schenberg-Frascino \& Moustacchi, 1972; Meyer et al., 1975; Lewis et al., 1993), high osmotic 
pressure (Mackenzie et al., 1986), and physical and chemical mutagens (Parry et al., 1976), than cells that are growing slowly or resting. Several factors may account for this variation in stress tolerance, given that cells undergo major physiological transitions between and within distinct growth phases in batch cultures. Several workers have reported the influence of factors such as plasma-membrane ATPase (Panaretou \& Piper, 1990; Rosa \& Sá-Correia, 1991), membrane composition (Thomas et al., 1978; Mishra \& Prasad, 1989), stressinduced proteins (Boucherie, 1985; Lindquist \& Craig, 1988; Bataillé et al., 1991; Collinson \& Dawes, 1992; Sanchez et al., 1992; Parsell et al., 1993), glycerol and other polyols (Brown et al., 1986; Reed et al., 1987), and trehalose (Gadd et al., 1987; Hottiger et al., 1987; Mackenzie et al., 1988; Attfield et al., 1992) on various stress tolerances of yeasts. It is highly likely that combinations of these and other factors are involved in tolerance to stress, and that their relative importance varies according to the type of stress and the physiological state of yeast cultures.

Trehalose is a storage disaccharide that is a potential factor in stress tolerance of yeasts. Studies show this sugat accumulates in S. cerevisiae (Attfield, 1987; Hottiger et al., 1987, 1989) and Schizosaccharomyces pombe (De Virgilio et al., 1990) during exposure to agents such as heat, alcohol, heavy metals and oxidants, indicating that it may play a part in inducible stress response in yeast. Whilst evidence is emerging to support this view (Hottiger et al., 1987, 1989; De Virgilio et al., 1990, 1991), it remains to be shown unequivocally that trehalose is a component of induced thermotolerance in cells growing exponentially on glucose (Panek et al., 1989, 1990).

There is limited evidence to suggest that intrinsic accumulation of trehalose is correlated with improved tolerance of lyophilization (Gadd et al., 1987) and hyperosmotic stress with glucose (Mackenzie et al., 1988), but these studies involved a single strain in the case of the former work, and the use of unrelated mutants in the latter. We previously described the generation of a series of related diploid strains of $S$. cerevisiae with varied capacities for accumulation of trehalose (Attfield et al., 1992). The trehalose-accumulation deficiency of these strains originated from a mutation designated cif 1 . The CIF1 gene is functionally related and apparently allelic to FDP, with both mapping close to LYS2 on chromosome II (Navon et al., 1979; Stucka \& Blázquez, 1993). These alleles share high sequence homology with TPS1 (Bell $e$ t al., 1992; González et al., 1992; Van Aelst et al., 1993), which encodes the $56 \mathrm{kDa}$ subunit of the trehalose-6phosphate synthase complex. Non-reverted or unsuppressed cif1 mutants are defective for growth on glucose, fructose or mannose, have impaired catabolite-inactivation of fructose-1,6-bisphosphatase, and are unable to accumulate trehalose (Van De Poll \& Schamhart, 1977; Navon et al., 1979; Panek et al., 1988). It therefore appears that CIF1, FDP and TPS1 are alleles which encode a protein that forms a crucial part of trehalose-6-phosphate synthase, whilst performing other functions essential to sensing and regulating glucose metabolism (Hohmann $e t$ al., 1993). The strains we generated previously (Attfield $e t$ al., 1992) exhibit restored ability to grow on glucose but still cannot accumulate trehalose. This partially reverted cif1 phenotype is similar to that exhibited by strains that carry the FPS ( $f d p$, cif1 suppressor) gene, which is nonallelic to FDP (Van Aelst $e t$ al., 1991).

This paper reports the use of our previously described strains to test the role of trehalose in intrinsic thermotolerance. We also report the construction of strains generated by crossing a yeast with the cif1-partially reverted phenotype (growth on glucose but greatly reduced trehalose accumulation) with isogenic haploids that have either functional CIF1 or disrupted cif $1:$ :HIS 3 genes. These strains were tested to determine whether CIF1 restores trehalose accumulation to cif1-partial revertants, and if so, whether this results in increased thermotolerance.

\section{METHODS}

Yeast strains, culture conditions, and assay of glucose and ethanol in culture supernatants. Strains used in this work are listed with genotypes in Table 1. Standard genetic methods were used in strain constructions (Sherman et al., 1981). Working cultures were streaked, grown at $30{ }^{\circ} \mathrm{C}$ for $3 \mathrm{~d}$ and maintained at $4{ }^{\circ} \mathrm{C}$ on GYP agar plates, which were $1 \%(\mathrm{w} / \mathrm{v})$ D-glucose, $1 \%$ $(\mathrm{w} / \mathrm{v})$ bacteriological peptone, $0.5 \%(\mathrm{w} / \mathrm{v})$ yeast extract, $0.3 \%$ $(\mathrm{w} / \mathrm{v})$ potassium dihydrogen orthophosphate, $0.005 \%(\mathrm{w} / \mathrm{v})$ adenine sulphate and $1.5 \%(\mathrm{w} / \mathrm{v})$ agar. Liquid medium, designated GY1/2P, was $2 \%$ D-glucose, $0 \cdot 5 \%$ bacteriological peptone, $0.5 \%$ yeast extract, $0.3 \%$ potassium dihydrogen orthophosphate and $0.005 \%$ adenine sulphate. All plate and liquid culture work involving haploid strain WDC-3A employed medium in which $\mathrm{D}$-glucose was replaced with $2 \%$ (w/v) D-galactose. Liquid cultures were grown at 180 r.p.m. and $30^{\circ} \mathrm{C}$ in conical shake flasks with liquid to air volume ratio of $1: 5$. Initial inocula were prepared by suspending three colonies of a strain in $10 \mathrm{ml} \mathrm{GY1/2P}$ and growing for $24 \mathrm{~h}$ (mid-respiratory growth phase). Subsequently, $2 \mathrm{ml}$ aliquots of these cultures were inoculated into $100 \mathrm{ml}$ of prewarmed $\left(30^{\circ} \mathrm{C}\right)$ and aerated $\mathrm{GY} 1 / 2 \mathrm{P}$ to provide experimental cultures. These were monitored regularly for glucose and ethanol concentrations in supernatants, after $1 \mathrm{ml}$ samples had been centrifuged for $30 \mathrm{~s}$ in Eppendorf tubes using a microfuge. Glucose was assayed by a glucose oxidase + horseradish peroxidase method (Yun \& Matheson, 1990). Ethanol was assayed by gas-liquid chromatography using a Varian 3300 series chromatograph with dual flame ionization detectors and a Varian 4400 series integrator. The glass column was $1.82 \mathrm{~m}$ long with an internal diameter of $6.5 \mathrm{~mm}$, and was packed with Porapak Q 80/100 mesh. Column temperature was $190^{\circ} \mathrm{C}$, injector $220^{\circ} \mathrm{C}$ and detector $220^{\circ} \mathrm{C}$. Carrier gas was nitrogen at $30 \mathrm{ml} \mathrm{min}^{-1}$; flame gases were hydrogen at $30 \mathrm{ml} \mathrm{min}$ and air at $300 \mathrm{ml} \mathrm{min}^{-1}$. Ethanol in culture supernatants was given by comparison with a known ethanol standard.

Stress conditions. Cells were heat-stressed at $52{ }^{\circ} \mathrm{C}$ following removal of $4 \mathrm{ml}$ culture from incubation medium and transfer to $22 \mathrm{~mm}$ diameter glass test tubes. Samples were heated rapidly to $50{ }^{\circ} \mathrm{C}$ in a $65{ }^{\circ} \mathrm{C}$ water bath before immediate transfer to a $52{ }^{\circ} \mathrm{C}$ water bath for various times. Samples were shaken occasionally during the treatment to keep cells suspended. Stress was relieved by removing $0.1 \mathrm{ml}$ portions to $0.9 \mathrm{ml}$ one-quarter-strength Ringer's solution (diluent) at room temperature $\left(22^{\circ} \mathrm{C}\right)$. Cells 
Table 1. Strains of S. cerevisiae used and their trehalose accumulation

Strains AH22 and DE6.1D are detailed in Attfield (1987) and Operti et al. (1982). Strains W303-1A and WDC-3A were kindly donated by M. A. Blázquez, Instituto de Investigaciones Biomédicas, Spain. Details of the construction and preliminary characterization of the strains designated by YN numbers are given by Attfield $e t$ al. (1992). PAY200 and PAY201 were constructed for this work using standard genetic methods (Sherman et al., 1981). All yeasts were sampled for trehalose at similar positions in their respiratory growth phase: sugar was exhausted and ethanol in culture supernatants was between $0.60 \%$ and $0.65 \%(\mathrm{w} / \mathrm{v})$.

\begin{tabular}{|c|c|c|}
\hline Strain no. & Genotype (phenotype) & $\begin{array}{l}\text { Trehalose } \\
\text { concn } \\
(\%, w / w)^{*}\end{array}$ \\
\hline \multicolumn{3}{|l|}{ Haploids } \\
\hline $\mathrm{AH} 22$ & a bis4 leu2 CIF1 & $4 \cdot 1 \pm 0 \cdot 31$ \\
\hline DE6.1D & $\alpha$ ade 2 lys 2 cif1(CPR for $\underline{\text { cif } 1}$ partial reversion) & $1 \cdot 0 \pm 0.67$ \\
\hline W303-1A & a ade 2 bis 3 ura 3 leu 2 trp 1 CIF 1 & $3 \cdot 2 \pm 0 \cdot 65$ \\
\hline WDC-3A & a ade 2 bis 3 ura 3 leu 2 trp 1 cif $1:: H I S 3$ & $1 \cdot 1 \pm 0 \cdot 38$ \\
\hline \multicolumn{3}{|l|}{ Diploids } \\
\hline YN150 & a/ $\alpha$ ADE/ade 2 bis4/HIS leu2/LEU LYS/lys2 CIF1/cif1(CPR) & $3 \cdot 8 \pm 0 \cdot 27$ \\
\hline YN154 & a $/ \alpha$ ADE/ade2 bis4/HIS leu2/LEU LYS/lys2 CIF1/cif1(CPR) & $4 \cdot 1 \pm 0.56$ \\
\hline YN155 & a/ $\alpha$ ADE/ade2 his4/HIS leu2/LEU LYS/lys2 CIF1/cif1(CPR) & $5 \cdot 0 \pm 0.58$ \\
\hline YN156 & a/ $\alpha$ ADE/ade2 bis4/HIS leu2/LEU CIF1/CIF1 & $6.4 \pm 0.46$ \\
\hline YN157 & a/ $\alpha$ bis4/HIS leu2/LEU LYS/lys2 CIF1/CIF1 & $5 \cdot 0 \pm 0 \cdot 33$ \\
\hline YN158 & $\mathrm{a} / \alpha$ ade $2 /$ ade 2 bis $4 /$ HIS leu $2 / L E U L Y S /$ lys 2 cif1(CPR)/cif1(CPR) & $0 \cdot 84 \pm 0.37$ \\
\hline YN159 & a $/ \alpha$ ade $2 /$ ade 2 bis $4 / H I S$ leu $2 / L E U ~ L Y S / l y s 2$ cif1(CPR)/cif1(CPR) & $0 \cdot 59 \pm 0 \cdot 21$ \\
\hline YN160 & $\mathrm{a} / \alpha$ ade $2 /$ ade 2 leu $2 / L E U L Y S /$ lys 2 cif1(CPR $) /$ cif1(CPR) & $0 \cdot 84 \pm 0 \cdot 24$ \\
\hline YN161 & a $/ \alpha A D E /$ ade 2 bis $4 / H I S$ leu $2 / L E U C I F 1 / C I F 1$ & $5 \cdot 4 \pm 0 \cdot 86$ \\
\hline YN162 & a/ $\alpha A D E /$ ade 2 bis4/HIS leu2/LEU CIF1/CIF1 & $7 \cdot 6 \pm 0 \cdot 84$ \\
\hline YN163 & a/a bis4/HIS leu2/LEU LYS/lys2 CIF1/CIF1 & $4.5 \pm 0.77$ \\
\hline YN164 & a/a bis4/HIS leu2/LEU LYS/lys2 CIF1/CIF1 & $6 \cdot 8 \pm 1 \cdot 21$ \\
\hline PAY200 & 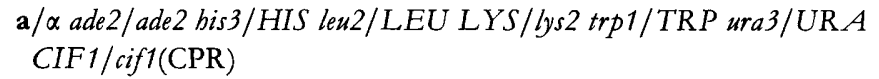 & $2 \cdot 7 \pm 0 \cdot 41$ \\
\hline PAY201 & $\begin{array}{l}\text { a/ } \alpha \text { ade } 2 / \text { ade } 2 \text { bis } 3 / \text { HIS leu2/LEU LYS/lys } 2 \operatorname{trp} 1 / T R P \text { ura } 3 / U R A \\
\text { cif } 1: \text { :HIS } 3 / \text { cif1(CPR) }\end{array}$ & $0 \cdot 94 \pm 0 \cdot 17$ \\
\hline
\end{tabular}

* Concentrations of trehalose accumulated in cells are means of at least three replicates $\pm \mathrm{SD}$.

were then diluted in diluent at room temperature and plated onto GYP agar. Plates were incubated for $5 \mathrm{~d}$ at $30^{\circ} \mathrm{C}$ prior to counting colonies. Survival was determined by comparison with viable counts of unstressed culture samples. Results shown are typical of those obtained in two to five repeated experiments. In all experiments, $100 \%$ viable counts of unstressed cultures ranged between $5 \times 10^{7}$ and $2 \times 10^{8}$ colony-forming units $\mathrm{ml}^{-1}$. Microscopic examination of cultures revealed no evidence of cell clumping before or after application of stress.

Extraction and assay of trehalose. Cells were harvested by centrifugation at $3000 \mathrm{~g}$ and room temperature for $5 \mathrm{~min}$. They were then resuspended in an equal volume of distilled water at $4{ }^{\circ} \mathrm{C}$ and re-centrifuged. This washing procedure was carried out three times. Cells were then sampled for dry weight, trehalose extracted with trichloroacetic acid and assayed by the anthrone method as described previously (Attfield, 1987). Analysis of extracts by thin-layer chromatography indicated that only trehalose was present as anthrone-positive material (Attfield, 1987).

\section{RESULTS}

\section{Intrinsic thermotolerances of related strains of $S$. cerevisiae that accumulate relatively high or low concentrations of trehalose}

The yeast strains designated by $\mathrm{YN}$ numbers as shown in Table 1 are not isogenic but they are closely related genetically. They were generated by crosses between strong or weak trehalose-accumulating haploids derived from YN150, or between those haploids and strains AH22 or DE6.1D, which were the haploid progenitors of YN150 (Attfield et al., 1992). Whereas AH22 is wild-type with respect to trehalose accumulation, strain DE6.1D is unable to accumulate high levels of the sugar (Operti et al., 1982; Attfield et al., 1992). The strains were grown in batch culture with glucose as the fermentative carbon source and tested during exponential respiro-fermentative (glucose-depletion and alcohol production) and respiratory (glucose exhausted and ethanol-depletion) growth 

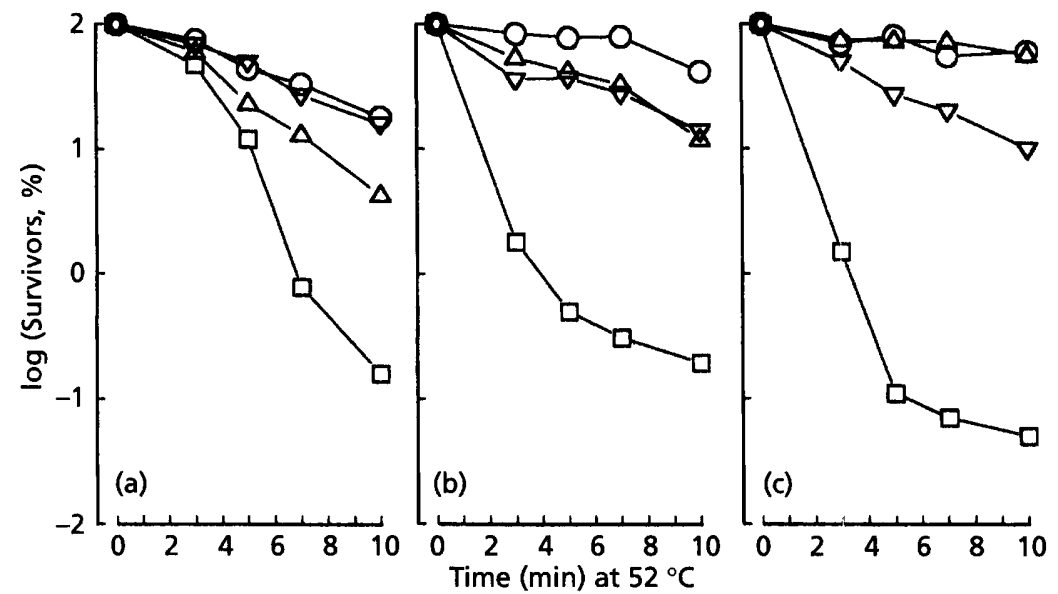

Fig. 1. Thermotolerances of $S$. cerevisiae strains that accumulate various levels of trehalose. Yeast were grown in batch culture at $30^{\circ} \mathrm{C}$ and 180 r.p.m. using GY1/2P medium; they were sampled in the respiratory phase (glucose depleted) when ethanol concentration was between $0.60 \%$ and $0.65 \%(\mathrm{w} / \mathrm{v})$, and exposed to $52{ }^{\circ} \mathrm{C}$ for the times indicated. Values in parentheses refer to trehalose contents of cells $(\%, w / w)$ at onset of stress application: (a) $O$, YN150 (3.8); $\triangle$, YN154 (4.1); $\nabla$, YN155 (5.0); $\square$ YN158 (0.84). (b) $\bigcirc$, YN156 (6.4); $\triangle$, YN157 (5.0); $\nabla, Y N 161$ (5.4); $\square$, YN159 (0.59). (c) 0 , YN162 (7.6); $\triangle$, YN164 (6.8); $\nabla$, YN163 (4.5); $\square$, YN160 (0.84)
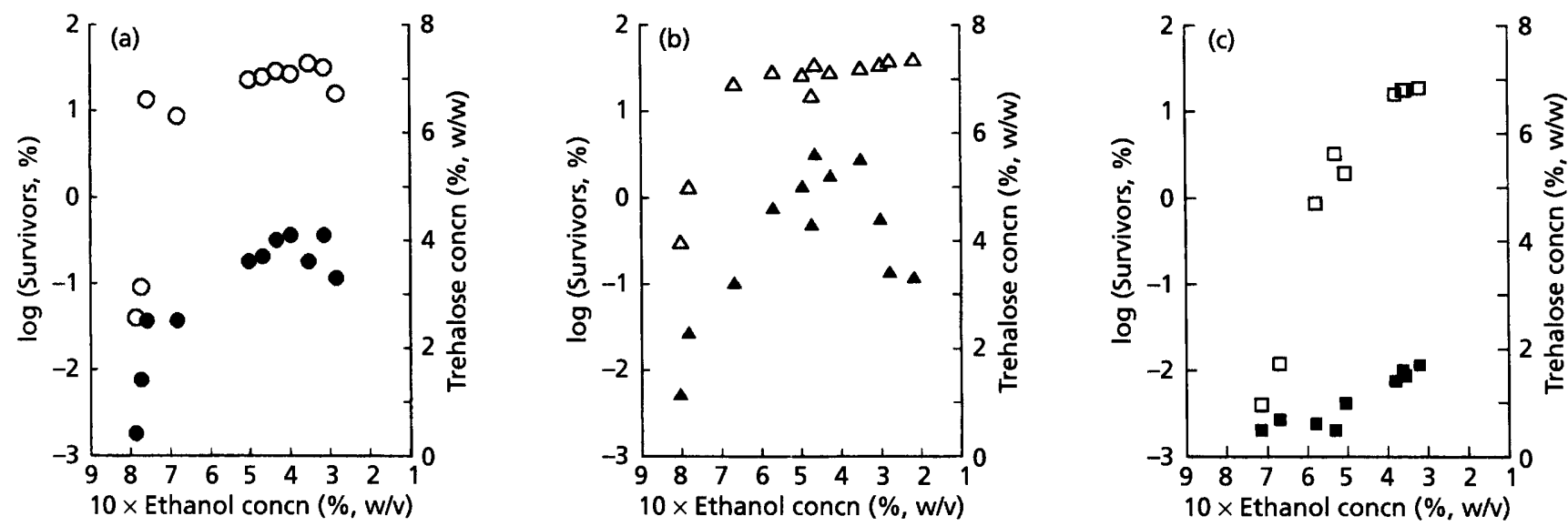

Fig. 2. Variations in thermotolerance and accumulated trehalose with respect to respiratory growth phase. Strains were batch cultured at $30^{\circ} \mathrm{C}$ and 180 r.p.m. using GY1/2P medium, and culture supernatants assayed for alcohol depletion during respiratory growth (glucose exhausted). Yeast were tested at the points indicated for trehalose concentration and tolerance to $52{ }^{\circ} \mathrm{C}$ stress for $15 \mathrm{~min}$. (a) $O$, YN150 survivors; $O, Y N 150$ trehalose; (b) $\triangle, Y N 164$ survivors; $\triangle, Y N 164$ trehalose; (c) $\square$, YN159 survivors; $\square$, YN159 trehalose.

phases (as defined by Lewis $e$ t al., 1993) for their intrinsic abilities to produce trehalose and to withstand $52^{\circ} \mathrm{C}$ heat stress. None of the strains accumulated trehalose above $0.7 \%(\mathrm{w} / \mathrm{w})$ and all were sensitive, with fewer than $0.1 \%$ surviving exposure to thermal stress during respirofermentative growth (data not shown). However, in early respiratory growth phase these same yeast strains showed marked differences in trehalose accumulation and heat tolerance. Strains YN158, YN159 and YN160 accumulated $<1 \%$ trehalose and were markedly more sensitive to thermal stress than the remaining strains, which stored $>3 \%$ of the disaccharide (Table 1, Fig. 1).

\section{Trehalose accumulation and thermotolerance throughout the respiratory growth phase}

Results reported by Lewis $e t$ al. (1993) indicate that stress tolerance can vary markedly for some yeast strains throughout their respiratory growth phases. Changes in internal concentrations of trehalose during this growth phase (Lillie \& Pringle, 1980; unpublished work of this laboratory) could be critical in this regard. Thermotolerance and trehalose accumulation in YN150 and YN164 (strong trehalose-accumulating strains) and YN159 (a weak trehalose-accumulating strain) were therefore followed closely with respect to respiratory growth phase. All three strains achieved similar cell densities $\left(5.5-7 \times 10^{7} \mathrm{ml}^{-\mathbf{1}}\right)$ and ethanol yields $(0.91-$ $0.93 \%, \mathrm{w} / \mathrm{v}$ ) by the end of respiro-fermentative growth on glucose and therefore had similar cultural conditions at the onset of respiratory phase. Although growth rates of the various strains on glucose and ethanol were very similar (data not shown), diminution of alcohol was measured, rather than time, to allow for accurate comparison of the different strains, and of replicate cultures of the same strains, at the same points within respiratory growth. In our experience there can be small, but nevertheless significant, day-to-day variations in times taken by cultures to proceed through different phases of batch growth. Such variations can be critical given that 

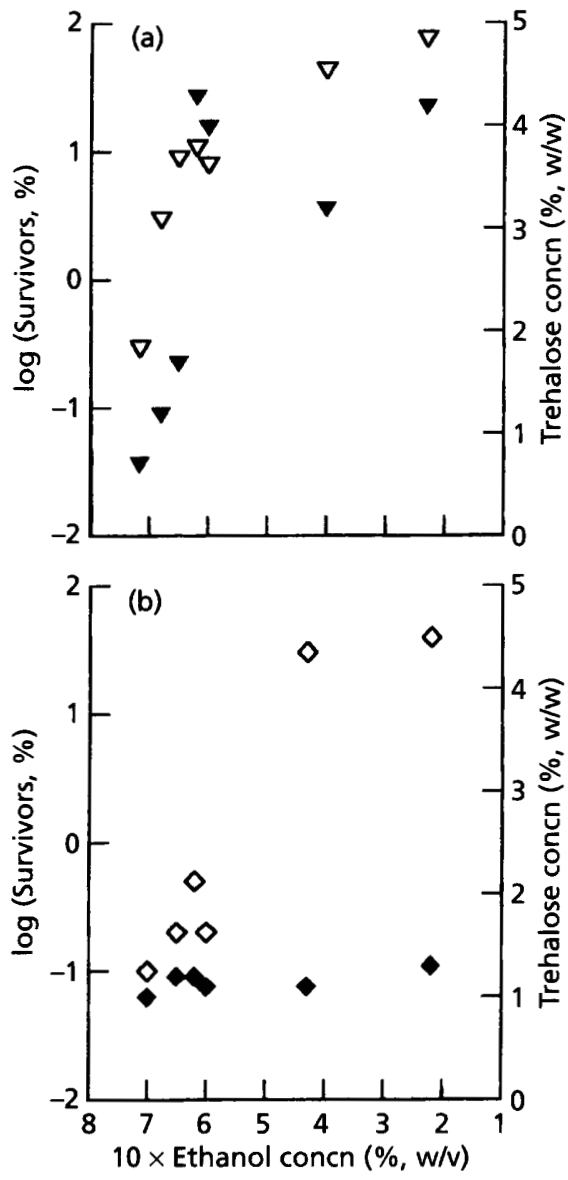

Fig. 3. Respiratory phase thermotolerance and accumulation of trehalose in isogenic strains with partially reverted cif1 phenotypes (CPR) and either functional or non-functional CIF1. Strains were grown and tested for trehalose and thermotolerance as described for Fig. 2, except that $52^{\circ} \mathrm{C}$ stress was applied for $30 \mathrm{~min}$. (a) $\nabla$, PAY200 CIF1/Cif1(CPR) survivors: $\nabla$, PAY200 CIF1/cif1(CPR) trehalose; (b) $\diamond$, PAY201 cif1::HIS3/cif1(CPR) survivors; $\diamond$, PAY201 cif1::HIS3/cif1(CPR) trehalose.

stress tolerances vary between and within different phases (Lewis et al., 1993) and so we favour the use of ethanol as a physiological measure of position within batch culture, rather than using the more traditional approach of time of culture.

Strains YN150 and YN164 stored trehalose rapidly and immediately at the onset of respiratory metabolism (Fig. $2 \mathrm{a}, \mathrm{b})$. Accumulation of trehalose continued in both strains until the later stages of the respiratory phase, where there was some, but not total, depletion of the disaccharide: trehalose remained at or above $3 \%(\mathrm{w} / \mathrm{w})$ in late respiratory phase (Fig. 2a, b). Thermotolerances of YN150 and YN164 paralleled trehalose accumulation in the early respiratory phase, but remained high at the later stage of this growth period, even when trehalose depletion was evident (Fig. 2a, b). YN159 showed relatively weak trehalose storage capacity. This strain never accumulated the disaccharide to the same extent as the other two strains and only exceeded $1 \%$ trehalose in the latter stages of respiratory growth (Fig. 2c). Thermotolerance appeared considerably more slowly in YN159 than in YN150 or YN164: the latter two strains produced $10 \%$ or more survivors and more than $2 \%$ trehalose by the time alcohol was depleted to approximately $0.7 \%(\mathrm{w} / \mathrm{v})$, whereas YN159 only produced about $0.01 \%$ survivors and $<1 \%$ $(\mathrm{w} / \mathrm{w})$ trehalose at the same stage (Fig. 2a-c). Strain YN159 did eventually achieve high levels of survivors considerably later in the respiratory phase $(>10 \%$ survivors when alcohol was depleted to $<0.4 \%, \mathrm{w} / \mathrm{v}$ ), but trehalose still did not exceed $2 \%$ (Fig. 2c).

\section{Construction and testing for trehalose accumulation and stress tolerance of isogenic diploid strains with a partially reverted cif1 phenotype and either functional or non-functional CIF1 alleles}

Even though all three strains that accumulated $<1 \%$ trehalose were more stress-sensitive than those that accumulated greater amounts of the sugar (Table 1, Fig. 1), the possibility exists that differences in stress tolerances are due to undefined factors in the strains, because the strains used in the above experiments were non-isogenic. Trehalose accumulation deficiency of strain DE6.1D is most likely to result from a cif1 mutation that is partially reverted or suppressed in this strain (Operti et al., 1982; Panek \& Bernades, 1983; Panek et al., 1988). The reversion confers low-level activity of trehalose-6-phosphate synthase and high activity of trehalase, with spontaneously restored ability to grow on glucose (Operti et al., 1982; unpublished work of this laboratory). We propose to term this phenotype CPR, for cif1 partial reversion. It is likely that diploid strains YN158, YN159 and YN160, which were formed by crossing DE6.1D with related haploids able to grow on glucose but unable to accumulate trehalose under conditions of nitrogen starvation (Attfield et al., 1992), are homozygous for a dominant partial reversion or suppression of $c i f 1$. If this is so, functional CIF1 should restore trehalose accumulation to DE6.1D. Moreover, the CPR phenotype of strain DE6.1D would be expected to alleviate deficiency for growth on glucose, but not restore trehalose accumulation proficiency to cif1 strains. In order to test this, strain DE6.1D cif1(CPR) was crossed with strain W303-1A CIF1 to yield PAY200 CIF1/cif1(CPR), and with an isogenic derivative of W303-1A, strain WDC-3A cif 1::HIS3, to give PAY201 cif1::HIS3/cif1(CPR). These two strains exhibit similar growth rates on glucose and ethanol (data not shown) and therefore PAY201 appears to be alleviated with respect to the glucose-growth deficiency associated with the cif $1:$ : HIS 3 mutation. However, this does not mean that all cif-related defects are alleviated by the CPR-phenotype, as is evidenced by the fact that this strain is deficient in trehalose accumulation, relative to its $C I F 1$ counterpart (Table 1 ).

During respiro-fermentative growth on glucose both PAY200 and PAY201 were equally hypersensitive to $52{ }^{\circ} \mathrm{C}$ and failed to store trehalose (data not shown). However, strain PAY200 showed rapid attainment of thermotolerance and accumulation of trehalose, whereas 
PAY201 exhibited weak trehalose storage and slow appearance of heat tolerance during early respiratory phase: PAY200 produced $10 \%$ survivors and $4 \%(\mathrm{w} / \mathrm{w})$ trehalose when alcohol was depleted to $0.6 \%(\mathrm{w} / \mathrm{v})$, whilst PAY201 gave $<1 \%$ survivors and $1 \cdot 1 \%$ trehalose at the same stage of culture (Fig. 3). The latter strain did, however show higher thermotolerance in later stages of respiratory growth (ethanol at $0.43 \%$ or less), even though trehalose remained below $2 \%$ at this time (Fig. 3). The PAY strains were innately more thermotolerant than the non-isogenic $\mathrm{YN}$ series of strains during respiratory growth phase, hence a $30 \mathrm{~min}$ exposure at $52{ }^{\circ} \mathrm{C}$ was used. Nevertheless, the results using the isogenic strains PAY200 and PAY201 were very similar in trend to those obtained using the non-isogenic series of yeasts.

\section{DISCUSSION}

Results from experiments using the related but nonisogenic CPR strains, and the isogenic CPR partners differing only with respect to full CIF1 functionality, suggest that CIF1 function is required, either directly or indirectly, for cells to develop intrinsic (uninduced by exogenous mild stress treatment) thermotolerance (Figs 1, 2 and 3). Intrinsic accumulation of trehalose and appearance of thermotolerance during early respiratory phase appeared closely linked in the non-isogenic strains YN150 and YN164 (Fig. 2). Similarly, studies with the isogenic CIF1/cif1(CPR) and cif1::HIS3/cif1(CPR) strains indicated that trehalose accumulation in early respiratory growth is concomitant with acquisition of intrinsic thermotolerance (Fig. 3). A possible interpretation of these findings is that the disaccharide acts in stress tolerance. Alternatively, it could be argued that the greater sensitivity to heat of strains YN158, 159 and 160 (Figs 1 and 2), and PAY201 cif1:: HIS3/cif1(CPR) (Fig. 3) is not due at all to trehalose-accumulation deficiency, but to one or more of the other possible effects of cif1 mutation in these strains, since even though growth on glucose is restored in the strains with CPR phenotype, not all cif1related deficiencies are corrected. Further characterization of the CIF1-related phenotypes of these CPR strains is in progress. Yet another interpretation of our data is that trehalose is a marker (Hottiger et al., 1992) appearing coincidently during intrinsic expression of stress tolerance factors, which are numerous (see, for example, the review by Piper, 1993). Currently, we argue that the weight of evidence supports the view that trehalose functions in thermotolerance. Not only is there increasing data from physiological studies on yeast (as demonstrated and cited in this paper), but also evidence from non-cellular systems. For example, trehalose has been shown to protect lipid bilayers and enzymes during freezing and thawing, drying and high temperature shifts (Rudolph \& Crowe, 1985; Crowe et al., 1987; Carpenter et al., 1988; Hottiger et al., 1994). These observations imply that trehalose could function actively in protection or repair processes rather than merely acting passively as a fermentable energy source for cells to access for repair processes. Further evidence to support this claim is provided by De Virgilio et al. (1994), who have described yeast mutants with inactivated cif1(tps1) or tps 2 genes (both encoding subunits of the trehalose-6-phosphate synthase/phosphatase complex) as failing to accumulate trehalose upon induction by mild heat-shock and exhibiting reduced thermotolerance relative to wild-type cells. Thus there is reasonably strong genetic and biochemical evidence of a role for trehalose in both intrinsic and inducible thermotolerance.

The extent to which intrinsically accumulated trehalose might function in thermotolerance appears to vary with respect to growth phase and status of cultures. Strain YN159 only achieved high thermotolerance $(>10 \%$ survivors with a maximum $<2 \%, \mathrm{w} / \mathrm{w}$, trehalose) in the latter stages of its respiratory phase, whereas strains YN150 and YN164 achieved equivalent thermotolerance at a more rapid rate, in concert with relatively high levels of the sugar $(>2 \%)$, much sooner in their respiratory phases (Fig. 2). Moreover YN150 and YN164 were not particularly thermotolerant very early in respiratory growth whilst trehalose was accumulated only to the low maximum level observed in strain YN159 at a much later stage of its respiratory growth (Fig. 2). These findings suggest the possibility that trehalose makes an important contribution to stress tolerance during early and midrespiratory growth phase, but that in the later stages, as the culture approaches stationary phase, other factors, which presumably YN159 is also capable of expressing, assume greater importance. The finding that trehalose diminished in YN150 and YN164 in the latter stages of the respiratory phase, but that these strains still retained high level thermotolerance at this time (Fig. 2), may also be indicative of other factors becoming more important for stress tolerance at this stage of culture. The fact that the isogenic CPR strains differing only with respect to functionality of CIF1 showed similar trends to the nonisogenic strains, with regard to rapid accumulation of trehalose and appearance of thermotolerance (Fig. 3), lends support to the concept that the sugar might be important for stress tolerance early in respiratory growth, but that its significance diminishes relative to other factors later in this growth phase.

Growth-phase-dependent variation in stress sensitivities of yeast cultures suggests that factors affecting tolerance are expressed intrinsically and variably. Sanchez et al. (1992) showed that the heat-shock gene HSP104 is expressed at higher basal levels in respiring cells than in fermenting cells and that it is involved in elevated intrinsic thermotolerance of respiring cells. Thus, it appears that the basal levels of expression of stress tolerance factors may vary according to growth phase and physiological status, and that this variation could influence the relative contributions of the different factors to stress tolerance during different stages of growth. It is worth noting that heat-inducible trehalose accumulation appears to be influenced by heat-shock proteins such as HSP70, HSP104 (Hottiger et al., 1992) and HSP90 (Cheng et al., 1993). Whether intrinsic accumulation of trehalose is affected by expression of these proteins in normally growing cells is not clear, although some heat-shock proteins are expressed variably and transiently throughout normal batch- 
culture of yeast (Boucherie, 1985; Werner-Washburne et al., 1989; Bataillé et al., 1991). The possibility therefore exists that the contribution of trehalose to intrinsic thermotolerance is influenced by the presence or absence of certain heat-shock proteins during normal growth.

The use of mild heat-shock or other non-lethal stresses to fully induce tolerance in cells may cause many stress tolerance factors to be expressed maximally. Whilst this generates high-level acquired stress tolerance, this artificial induction approach may mask the subtleties of relative contributions from various factors involved in cells growing in normal batch-culture. It is likely that cells are stressed during aerobic batch-culture as they undergo a major shift in metabolic processes from essentially fermentation on glucose to full respiratory and slower growth on alcohol. It may be that relative intrinsic expression of stress-response genes is a reaction to the stress level perceived by a cell as it shifts metabolism, and that if a cell is lacking a particular tolerance function the expression of other stress genes could be enhanced. Thus, if a cell is capable of overcoming a particular level of stress by, for example, employing trehalose as a defence, this may reduce the need for involvement of other factors. Conversely, if a cell is incapable of producing trehalose to a significant level, it may be more reliant upon other stress tolerance factors that could be expressed more strongly than usual. This could explain why strains YN159 and PAY201 cif1::HIS3/cif1(CPR) achieved thermotolerance late in the respiratory phase even though they accumulated only low levels of trehalose. We are currently testing the relative levels of expression of various stress genes in these strains in an attempt to clarify this. Hence, whilst we favour the view that trehalose is important as an intrinsic stress tolerance factor, at least in early respiratory phase cells, it can only be considered as one of many determinants of stress tolerance. Certainly numerous factors determine stress tolerance (Piper, 1993), and many of these may interact.

Clearly, it is desirable ultimately to separate trehalose effects from all other possible functions of CIF1, and the other numerous stress tolerance factors available to the cell, when trying to determine, unequivocally, a role for the disaccharide in stress tolerance. However even this may not be sufficient because the sugar appears to have important functions in carbon metabolism that may be additional to its potential direct role in stress tolerance. For example it may function in regulation of glycolytic flux (Lillie \& Pringle, 1980; Panek, 1985; Hohmann et al., 1993). Thus any manipulation of trehalose metabolism may inadvertently affect stress tolerance, not just because of a possible involvement of the sugar in protecting proteins and lipids, but also because of its apparent function in regulation of central carbon metabolism.

\section{ACKNOWLEDGEMENTS}

The authors are grateful to the management of Burns Philp R\&D Pty Ltd for their kind permission to publish this work. We especially thank Miguel Blázquez for kindly providing strains.

\section{REFERENCES}

Attfield, P. V. (1987). Trehalose accumulates in Saccharomyces cerevisiae during exposure to agents that induce heat shock response. FEBS Lett 225, 259-263.

Attfield, P. V., Raman, A. \& Northcott, C. J. (1992). Construction of Saccharomyces cerevisiae strains that accumulate relatively low concentrations of trehalose, and their application in testing the contribution of the disaccharide to stress tolerance. FEMS Microbiol Lett 94, 271-276.

Bataillé, N., Régnacq, M. \& Boucherie, H. (1991). Induction of a heat-shock-type response in Saccharomyces cerevisiae following glucose limitation. Yeast 7, 367-378.

Bell, W., Klaassen, P., Ohnacker, M., Boller, T., Herweijer, M., Schoppink, P., Van Der Zee, P. \& Wiemken, A. (1992). Characterisation of the $56 \mathrm{kDa}$ subunit of yeast trehalose-6-phosphate synthase and cloning of its gene reveal its identity with the product of CIF1, a regulator of carbon catabolite inactivation. Eur J Biochem 209, 951-959.

Boucherie, H. (1985). Protein synthesis during transition and stationary phases under glucose limitation in Saccbaromyces cerevisiae. J Bacteriol 161, 385-392.

Brown, A. D., Mackenzie, K. F. \& Singh, K. K. (1986). Selected aspects of microbial osmoregulation. FEMS Microbiol Rev 39, 31-36.

Carpenter, J. F., Martin, B., Loomis, S. H. \& Crowe, J. H. (1988). Long-term preservation of dried phosphofructokinase by sugars and sugar/zinc mixtures. Cryobiology 25, 372-376.

Cheng, L., Kirk, N. \& Piper, P. (1993). A small influence of HSP90 levels on the trehalose and heat shock element inductions of the yeast heat shock response. Biochem Biopbys Res Commun 195, 201-207.

Collinson, L. P. \& Dawes, I. W. (1992). Inducibility of the response of yeast cells to peroxide stress. J Gen Microbiol 138, 329-335.

Crowe, J. H., Crowe, L. M., Carpenter, J. F. \& Aurell-Wistrom, C. (1987). Stabilisation of dry phospholipid bilayers and proteins by sugars. Biocbem J 242, 1-10.

De Virgilio, C., Hottiger, T., Dominguez, J., Boller, T. \& Wiemken, A. (1994). The role of trehalose synthesis for the acquisition of thermotolerance in yeast. I. Genetic evidence that trehalose is a thermoprotectant. Eur J Biochem 219, 179-186.

De Virgilio, C., Simmen, U., Hottiger, T., Boller, T. \& Wiemken, A. (1990). Heat shock induces enzymes of trehalose metabolism, trehalose accumulation, and thermotolerance in Schizosaccharomyces pombe, even in the presence of cycloheximide. FEBS Lett 273, 107-110.

De Virgilio, C., Piper, P., Boller, T. \& Wiemken, A. (1991). Acquisition of thermotolerance in Saccharomyces cerevisiae without heat shock protein hsp104 and in the absence of protein synthesis. FEBS Lett 288, 86-90.

Gadd, G. M., Chalmers, K. \& Reed, R. H. (1987). The role of trehalose in dehydration resistance of Saccharomyces cerevisiae. FEMS Microbiol Lett 48, 249-254.

González, M. I., Stucka, R., Blázquez, M. A., Feldmann, H. \& Gancedo, C. (1992). Molecular cloning of CIF1, a yeast gene necessary for growth on glucose. Yeast 8, 183-192.

Hohmann, S., Neves, M. J., de Koning, W., Alijo, R., Ramos, J. \& Thevelein, J. M. (1993). The growth and signalling defects of the ggs 1 ( $f d p 1 /$ byp 1$)$ deletion mutant on glucose are suppressed by a deletion of the gene encoding hexokinase PII. Curr Genet 23, 281-289.

Hottiger, T., Boller, T. \& Wiemken, A. (1987). Rapid changes of 
heat and desiccation tolerance correlated with changes of trehalose content in Saccharomyces cerevisiae cells subjected to temperature shifts. FEBS Lett 220, 113-115.

Hottiger, T., Boller, T. \& Wiemken, A. (1989). Correlation of trehalose content and heat resistance in yeast mutants altered in the RAS/adenylate cyclase pathways: is trehalose a thermoprotectant? FEBS Lett 255, 431-434.

Hottiger, T., De Virgilio, C., Bell, W., Boller, T. \& Wiemken, A. (1992). The 70-kilodalton heat-shock proteins of the SSA subfamily negatively modulate heat-shock-induced accumulation of trehalose and promote recovery from heat stress in the yeast, Saccharomyces cerevisiae. Eur J Biochem 210, 125-132.

Hottiger, T., De Virgilio, C., Hall, M. N., Boller, T. \& Wiemken, A. (1994). The role of trehalose synthesis for the acquisition of thermotolerance in yeast. II. Physiological concentrations of trehalose increase the thermal stability of proteins in vitro. Eur $J$ Biochem 219, 187-193.

Lewis, J. G., Northcott, C. J., Learmonth, R. P., Attfield, P. V. \& Watson, K. (1993). The need for consistent nomenclature and assessment of growth phases in diauxic cultures of Saccharomyces cerevisiae. J Gen Microbiol 139, 835-839.

Lillie, S. H. \& Pringle, J. R. (1980). Reserve carbohydrate metabolism in Saccharomyces cerevisiae : responses to nutrient limitation. J Bacteriol 143, 1384-1394.

Lindquist, S. \& Craig, E. A. (1988). The heat shock proteins. Annu Rev Genet 22, 631-637.

Mackenzie, K. F., Blomberg, A. \& Brown, A. D. (1986). Water stress plating hypersensitivity of yeasts. $J$ Gen Microbiol 132, 2053-2056.

Mackenzie, K. F., Singh, K. K. \& Brown, A. D. (1988). Water stress plating hypersensitivity of yeasts: protective role of trehalose in Saccharomyces cerevisiae. J Gen Microbiol 134, 1661-1666.

Meyer, E. D., Sinclair, N. A. \& Nagy, B. (1975). Comparison of the survival and metabolic activity of psychrophilic and mesophilic yeasts subjected to freeze-thaw stress. Appl Microbiol 29, 739-744.

Mishra, P. \& Prasad, R. (1989). Relationship between ethanol tolerance and fatty acyl composition of Saccharomyces cerevisiae. Appl Microbiol Biotechnol 30, 294-298.

Navon, G., Shulman, R. G., Yamane, T., Eccleshall, T. R., Lam, K. B., Baronfsky, J. J. \& Marmur, J. (1979). Phosphorous-31 nuclear magnetic resonance studies of wild type and glycolytic pathway mutants of Saccharomyces cerevisiae. Biochemistry 18, 4487-4499.

Operti, M. S., Oliveira, D. E., Freitas-Valle, A. B., Oestreicher, E. G., Mattoon, J. R. \& Panek, A. D. (1982). Relationships between trehalose metabolism and maltose utilisation in Saccharomy'es cerevisiae. Curr Genet 5, 69-76.

Panaretou, B. \& Piper, P. W. (1990). Plasma-membrane ATPase action affects several stress tolerances of Saccharomyces cerevisiae and Schizosaccharomyces pombe as well as the extent and duration of the heat shock response. J Gen Microbiol 136, 1763-1770.

Panek, A. C., Francois, J. \& Panek, A. D. (1988). New insights into a mutant of Saccharomyces cerevisiae having impaired sugar uptake metabolism. Curr Genet 13, 15-20.

Panek, A. C., Mansure Vânia, J. J., Paschoalin, M. F. \& Panek, A. D. (1990). Regulation of trehalose metabolism in Saccharomyces cerevisiae mutants during temperature shifts. Biochimie 72, 77-79.

Panek, A. D. (1985). Trehalose metabolism and its role in Saccharomyces cerevisiae. J Biotechnol 3, 121-130.

Panek, A. D. \& Bernades, E. J. (1983). Trehalose: its role in germination of Saccharomyces cerevisiae. Curr Genet 7, 393-397.
Panek, A. D., Ferreira, R. \& Panek, A. C. (1989). Comparative studies between the glucose-induced phosphorylation signal and the heat shock response in mutants of Saccharomyces cerevisiae. Biochimie 71, 313--318.

Parry, J. M., Davies, P. J. \& Evans, W. E. (1976). The effects of " cell age" upon the lethal effects of chemical and physical mutagens in the yeast Saccharomyces cerevisiae. Mol \& Gen Genet 146, 27-35.

Parsell, D. A., Taulien, J. \& Lindquist, S. (1993). The role of heatshock proteins in thermotolerance. Philosoph Trans Roy Soc Lond B 339, 279-286.

Piper, P. W. (1993). Molecular events associated with acquisition of heat tolerance by the yeast Saccharomyces cerevisiae. FEMS Microbiol Rev 11, 339-356.

Reed, R. H., Chudek, J. A., Foster, R. \& Gadd, G. M. (1987). Osmotic significance of glycerol accumulation in exponentially growing yeasts. Appl Environ Microbiol 53, 2119-2123.

Rosa, M. F. \& Sá-Correia, I. (1991). In vivo activation by ethanol of plasma membrane ATPase of Saccharomyces cerevisiae. Appl Environ Microbiol 57, 830-835.

Rudolph, A. S. \& Crowe, J. H. (1985). Membrane stabilisation during freezing: the role of two natural cryoprotectants, trehalose and proline. Cryobiology 22, 367-377.

Sanchez, Y., Taulien, J., Borkovich, A. \& Lindquist, S. (1992). Hsp 104 is required for tolerance to many forms of stress. EMBO J 11, 2357-2364.

Schenberg-Frascino, A. \& Moustacchi, E. (1972). Lethal and mutagenic effects of elevated temperature on haploid yeast. $\mathrm{Mol} \&$ Gen Genet 115, 243-257.

Sherman, F., Fink, G. R. \& Hicks, J. B. (1981). Methods in Yeast Genetics. Cold Spring Harbor, NY: Cold Spring Harbor Laboratory.

Stucka, R. \& Blázquez, M. A. (1993). The $f d p 1$ and cif1 mutations are caused by different single nucleotide changes in the yeast CIF1 gene. FEMS Microbiol Lett 107, 251-254.

Thomas, S. D., Hossack, J. A. \& Rose, A. H. (1978). Plasmamembrane lipid composition and ethanol tolerance in Saccharomyces cerevisiae. Arch Microbiol 117, 239-245.

Van Aelst, L., Hohmann, S., Zimmermann, F. K., Jans, A. W. H. \& Thevelein, J. M. (1991). A yeast homologue of the bovine lens fibre MIP gene family complements the growth defect of a Saccharomyces cerevisiae mutant on fermentable sugars but not its defect in glucoseinduced RAS-mediated cAMP signalling. EMBO J 10, 2095-2104.

Van Aelst, L., Hohmann, S., Bulaya, B., de Koning, W., Sierkstra, L., Neves, M. J., Luyten, K., Alijo, R., Ramos, J., Coccetti, P., Martegani, E., de Magalhães-Rocha, N. M., Brandão, R. L., Van Dijck, P., Vanhalewyn, M., Durnez, P., Jans, A. W. H. \& Thevelein, J. M. (1993). Molecular cloning of a gene involved in glucose sensing in the yeast Saccharomyces cerevisiae. Mol Microbiol 8, 927-943.

Van De Poll, K. W. \& Schamhart, D. H. J. (1977). Characterisation of a regulatory mutant of fructose 1,6-bisphosphatase in $\mathrm{Sac}$ charomyces carlsbergensis. Mol \& Gen Genet 154, 61-66.

Werner-Washburne, M., Becker, J., Kosic-Smithers, J. \& Craig, E. A. (1989). Yeast Hsp70 RNA levels vary in response to the physiological status of the cell. J Bacteriol 171, 2680-2688.

Yun, S-K \& Matheson, N. K. (1990). Estimation of amylose content of starches after precipitation of amylopectin by conconavalin-A. Starch/Stärke 42, 302-305.

Received 10 March 1994; revised 15 May 1994; accepted 25 May 1994. 